\title{
Bayesian Edge Regularization in Range Image Segmentation
}

\author{
Smaine Mazouzi ${ }^{1}$ and Mohamed Batouche ${ }^{2}$ \\ ${ }^{1}$ LERI-CReSTIC, Université de Reims, B.P. 1035, 51687, Reims, France \\ mazouzi@leri.univ-reims.fr \\ ${ }^{2}$ Département d'informatique, Université de Constantine, 25000, Algérie \\ batouche@wissal.dz
}

\begin{abstract}
We present in this paper a new method for improving range image segmentation, based on Bayesian regularization of edges produced by an initial segmentation. The method proceeds in two stages. First, an initial segmentation is produced by a randomized region growing technique. The produced segmentation is considered as a degraded version of the ideal segmentation, which should be then refined. In the second stage, image pixels not labeled in the first stage are assigned to the resulting regions by using a Bayesian estimation based on some prior assumptions on the region boundaries. The image priors are modeled by a new Markov Random Field (MRF) model. Contrary to most of the authors in range image segmentation, who use surface smoothness MRF models, our MRF model is based on the smoothness of region boundaries, used to improve the initial segmentation by a Bayesian regularization of the resulting edges. Tests performed with real images from the ABW database show a good potential of the proposed method for significantly improving the segmentation results.
\end{abstract}

Keywords: Image Segmentation, Range Image, Randomized Region Growing, Bayesian Estimation, Markov Random Field.

\section{Introduction}

Segmenting an image consists in assigning its pixels in homogenous, continuous, and disjoint sets, called image regions. Image segmentation is often essential for high level image analysis and understanding. In an image, a region is defined as the set of contiguous pixels that share a common propriety, called the region homogeneity criterion. In range images, segmentation methods can be divided in two distinct categories: edge-based segmentation methods and region-based segmentation methods. In the first category, pixels which correspond to discontinuities in depth or in surface normals are selected and chained in order to delimit the regions in the image [511]. Edge-based methods are well known for their low computational cost; however they are very sensitive to noise. Regionbased methods use geometrical surface proprieties to gather pixels with the same properties in disjoint regions 41]. Compared to edge-based methods, they are more stable and less sensitive to noise. However, their efficiency depends strongly on the selection of the region seeds. 
Few authors have integrated Bayesian inference in range image segmentation. Jain and Nadabar 9] have proposed a Bayesian method for edge detection in range images. Authors use the Line Process (LP) Markov Random Field (MRF) model [7] to label image pixels as EDGE or NON-EDGE pixels. Wang and Wang [15] have presented a hybrid scheme for range image segmentation. First, they proposed a joint Bayesian estimation of both pixel labels, and surface patches. Next, the solution is improved by combining the Scan Line algorithm [1], and the Multi-Level Logistic (MLL) MRF model [14]. In spite of various contributions of the works previously cited, some aspects inherent to range image segmentation were omitted. Indeed, most of the works use markovian models that are based exclusively on the surface smoothness prior. Moreover, the proposed methods proceed by assigning pixels to clusters without ensuring the continuity of the resulting clusters. Typically, in the approach proposed by Wang and Wang [15], pixels belonging to coplanar regions may be labeled equally in any of these regions. The spatial continuity constraint of resulting regions seems that it was not taken into account.

The approach proposed in this paper provides first an initial segmentation version, using an improved region growing technique, and then refines this version by a Bayesian-MRF labeling. The refinement of the initial segmentation consists in a Bayesian regularization of unlabeled pixels. The latter are mostly close to region boundaries. A new Markov random field model is used to model the prior information on region boundaries, assuming that edges in range images are piecewise smooth. The new MRF model uses a high-order neighborhood system, and is based on the assumption that edge pixels are situated on straight lines that represent region boundaries. The use of the ICM algorithm (Iterated Conditional Modes) 3] to search for the optimal solution has allowed us to formulate region continuity by defining a constraint on the possible labels of a given pixel. The experimentations performed with real images from the ABW database [8] show a good potential of the proposed method to improve results in range image segmentation.

The remainder of the paper is organized as follows: In Section 2, we introduce the image segmentation by randomized region growing. Section 3 is devoted to the proposed Bayesian approach for segmentation refinement. We present in this section the new Markov Random Field (MRF) model, as well as the adaptation of the ICM algorithm for the search of the optimal solution. Experimentations are shown in Section 4, in which we present respectively the evaluation framework, parameter selection, and some experimental results. Finally, a conclusion summarizes our contribution.

\section{Segmentation by Randomized Region Seed Sampling}

A range image is a discretized two-dimensional array where at each pixel $(x, y)$ is recorded the distance $d(x, y)$ between the range finder plane and the corresponding point of the scene. In order to define a homogeneity criterium allowing region growing, we use a new representation $\left(d^{*}\right)$ of the row image, where $d^{*}(x, y)$ 
represents the tangent plane to the surface at $(x, y)$. The tangent plane at $(x, y)$ is obtained by the multiple regression method using the set of neighboring pixels situated within a $3 \times 3$ window centred at $(x, y)$, and whose depths are close, according to a given threshold $\left(T r_{h}\right)$. The plane equation in a $3-D$ coordinate system may be expressed as follows:

$$
z=a x+b y+c
$$

where $(a, b,-1)^{T}$ is a normal vector to the plane, and $|c| / \sqrt{a^{2}+b^{2}+1}$ is the orthogonal distance between the plane and the coordinate origin. We consider that a pixel belongs to a planar region, given its plane equation, if the distance between the respective planes is less than $\left(T r_{h}\right)$, and the angle between the respective normals is less than $\operatorname{Tr}_{\theta}$, where $T r_{h}$ and $\operatorname{Tr}_{\theta}$ are respectively the distance and the angle thresholds. The quality of plane estimation $q(x, y)$ at $(x, y)$ according to the regression model is also computed. The latter is used to select the best region seeds in the region growing algorithm.

Inspired from the RANSAC algorithm [6], our region growing technique is based on random sampling of the region seeds. A generated seed is accepted if only the surface estimation quality $q$ at this seed is greater than a given threshold $Q$. For every accepted seed, a region growing is performed by recursively including homogenous pixels situated on the borders the region in growth. A given seed centred at $\left(x_{t}, y_{t}\right)$ is formed by the pixels in a $W \times W$ window, belonging to the same plane. The seed quality is represented by the minimum of estimation qualities of pixels that form the seed. Selection-growing process is repeated until no new region can be created. The randomized growing algorithm is described as follows:

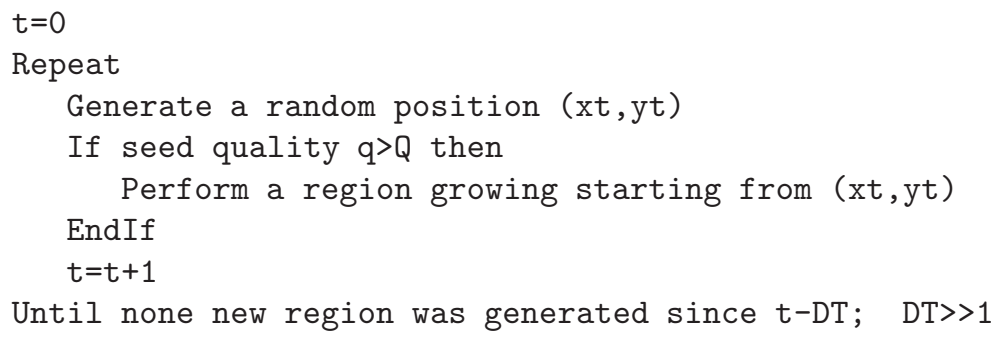

Random sampling of region seeds permits to select the best seeds. These latter are characterized by a good quality. It allows to include in a given region the largest possible set of homogenous pixels. Indeed, several seeds within the same region can be generated; however none of these seeds is accepted. The first generated seed for which the quality $q$ is greater than $Q$ will be accepted and considered for region growing.

For each generated region $R_{l}$, the residual variance $\sigma_{l}^{2}$ is calculated as follows:

$$
\sigma_{l}^{2}=\sum_{(x, y) \in R_{l}}\left(a_{l} x+b_{l} y+c_{l}-d(x, y)\right)^{2}
$$

where $\left(a_{l}, b_{l}, c_{l}\right)$ are the plane parameters of the region $R_{l}$. 


\section{Edge Regularization as Bayesian Estimation}

\subsection{Bayesian-MRF Pixel Labeling}

We have used the piecewise smoothness of region boundaries as prior to model distributions of pixel labels $(\mathrm{MRF})$ in range images. Let $S$ denote the image lattice. At each site $(x, y) \in S, d(x, y)$ is the depth at the site, and $d^{*}(x, y)$ represents the corresponding tangent plane equation parameters: $d^{*}(x, y)=$ $\left(a_{x, y}, b_{x, y}, c_{x, y}\right)$. Let $M$ be the number of regions in the image. So, each site $(x, y)$ can take a label $f_{x, y}$ from the set of labels $L=\left\{l_{1}, \ldots, l_{M}\right\}$. The labeling set $F=\left\{f_{x, y},(x, y) \in S, f_{x, y} \in L\right\}$, represents a segmentation of the image. If we assume that $F$ is markovian, segmenting $S$ according to the Bayesian-MRF framework [14] can be done by calculating the maximum a posteriori (MAP) of the distribution of the set $F: P(F / d)$, by considering $F$ as a Markov Random Field (MRF).

According to Bayes' rule, the maximum a posteriori $P(F / d)$ is expressed as follows:

$$
P(F / d)=\frac{p(d / F) P(F)}{p(d)}
$$

$P(F)=Z^{-1} e^{-U(F)}$ is the a priori probability of $F$ obtained according to the Markov-Gibbs equivalence theorem [2].

$Z=\sum_{F} e^{-U(F)}$ is a normalization constant called the partition function. The a priori energy $U(F)$ is a sum of clique potentials $V_{c}(F)$ over the set of all possible cliques $C: U(F)=\sum_{c \in C} V_{c}(F)$.

In order to model the edge smoothness we use cliques formed by 9 sites located in a $3 \times 3$ window. Let $c_{9}$ be a clique of $3 \times 3$ sites centred at $(x, y)$, and let $\zeta$ $(\zeta<0)$ a potential parameter. Considering possible configurations in Fig. 1, the potential $V_{c}$ of cliques in $C$ can be expressed as follows:

$$
V_{c}\left(c_{9}(x, y)\right)=\left\{\begin{array}{l}
\zeta \text { if } \exists\left(x^{\prime}, y^{\prime}\right),\left(x^{\prime \prime}, y^{\prime \prime}\right) \mid f_{x, y}=f_{x^{\prime}, y^{\prime}}=f_{x^{\prime \prime}, y^{\prime \prime}} \\
\text { and } \phi\left(\left(x^{\prime}, y^{\prime}\right),(x, y),\left(x^{\prime \prime}, y^{\prime \prime}\right)\right)=\pi \\
0 \text { if } \exists\left(x^{\prime}, y^{\prime}\right),\left(x^{\prime \prime}, y^{\prime \prime}\right) \mid f_{x, y}=f_{x^{\prime}, y^{\prime}}=f_{x^{\prime \prime}, y^{\prime \prime}} \\
\text { and } \phi\left(\left(x^{\prime}, y^{\prime}\right),(x, y),\left(x^{\prime \prime}, y^{\prime \prime}\right)\right)=2 \pi / 3 \\
-\zeta \text { otherwise }
\end{array}\right.
$$

where $\phi\left(\left(x^{\prime}, y^{\prime}\right),(x, y),\left(x^{\prime \prime}, y^{\prime \prime}\right)\right)$ is the angle between the two vectors $\left(x^{\prime}-x, y^{\prime}-\right.$ $y)^{T}$ and $\left(x^{\prime \prime}-x, y^{\prime \prime}-y\right)^{T}$.

Configurations used to define $V_{c}$ depend on the surface type. For images containing polyhedral objects, considered in this work, $V_{c}$ is defined on the basis that the boundary between two adjacent regions is formed by pixels belonging to the same straight line (Fig. 11). So, configurations which correspond to locally unsmoothed edges are penalized by using a positive clique potential $(-\zeta)$.

The likelihood distribution $p(d / F)$, is obtained by assuming that the observations $d$ are degraded by an independent Gaussian noise: $d(x, y)=a_{f_{x, y}} x+$ 
$b_{f_{x, y}} y+c_{f_{x, y}}+e(x, y)$, with $e(x, y) \sim N\left(0, \sigma_{f_{x, y}}^{2}\right)$. So, the likelihood distribution is expressed as follows:

$$
p(d / F)=\frac{1}{\prod_{(x, y) \in S} \sqrt{2 \pi \sigma_{f_{x, y}}^{2}}} e^{-U(d / F)}
$$

with the likelihood energy $U(d / F)$ defined by:

$$
U(d / F)=\sum_{(x, y) \in S}\left(a_{f_{x, y}} x+b_{f_{x, y}} y+c_{f_{x, y}}-d(x, y)\right)^{2} / 2 \sigma_{f_{x, y}}^{2}
$$

Since $p(d)$ is constant for a fixed $d$, the solution $F^{*}$ is obtained by maximizing the a posteriori probability $P(F / d) \propto p(d / F) P(F)$, which is equivalent to minimizing the a posteriori energy $U(F / d)=U(d / F)+U(F): F^{*}=\operatorname{argmin}\{U(d / F)+$ $U(F)\}$.

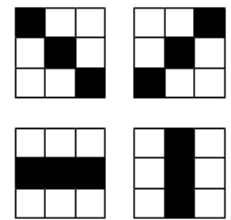

(a)

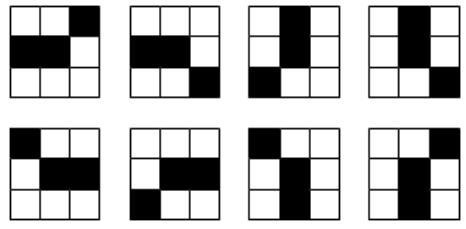

(b)

Fig. 1. Clique potential $V_{c}\left(c_{9}\right)$ defined according to the edge smoothness prior. (a) Full locally smooth edge : $V_{c}\left(c_{9}\right)=\zeta$; (b) Partial locally smooth edge : $V_{c}\left(c_{9}\right)=0$; Otherwise, the edge is not locally smooth : $V_{c}\left(c_{9}\right)=-\zeta$.

\subsection{Searching for the Optimal Solution}

By assuming that $F$ is markovian, and the observations $\{d(x, y) ;(x, y) \in S\}$ are conditionally independent, we have used the ICM algorithm [3] to minimize the a posteriori energy $U(F / d)$. By considering $U(F / d)$ as a sum of energies over all image sites: $U(F / d)=\sum_{(x, y) \in S} U\left(f_{x, y} / d(x, y)\right)$, we can separate it in two terms:

$$
U(F / d)=\sum_{(x, y) \in S^{\prime}} U\left(f_{x, y} / d(x, y)\right)+\sum_{(x, y) \in S-S^{\prime}} U\left(f_{x, y} / d(x, y)\right)
$$

where $S^{\prime}$ is the set of sites which have not been labeled in the first stage (by region growing): $S^{\prime}=\left\{(x, y) \in S \mid f_{x, y}\right.$ is undefined $\}$. Assuming the correctness of the labeling of the set $S-S^{\prime}$ (performed in the first stage), the term $\sum_{(x, y) \in S-S^{\prime}} U\left(f_{x, y} / d(x, y)\right)$ is thus constant. Minimizing the energy $U(F / d)$ is equivalent to minimizing the energy $U^{\prime}(F / d)$ which corresponds to the sites in $S^{\prime}$ :

$$
U^{\prime}(F / d)=\sum_{(x, y) \in S^{\prime}} U\left(f_{x, y} / d(x, y)\right)
$$


The assumption of the correctness of the labeling of $S-S^{\prime}$ allows also to define a constraint on the set of possible values that a site in $S^{\prime}$ can have during the execution of the ICM algorithm. Indeed, the label $f_{x, y}^{k}$ at the iteration $k$, of a site $(x, y)$ is chosen among the set $L^{\prime}(x, y) \subset L$ containing the labels of the sites labeled in the first stage, and located in a $W \times W$ window centred at $(x, y)$. Formally, $L^{\prime}(x, y)$ is defined as follows:

$$
L^{\prime}(x, y)=\left\{l \mid \exists\left(x^{\prime}, y^{\prime}\right) \in S-S^{\prime},\left(x^{\prime}-x, y^{\prime}-y\right) \in[-W / 2, W / 2]^{2} \wedge f_{x^{\prime}, y^{\prime}}=l\right\}
$$

The previous heuristics allow to speed up the calculation of the minimum of the a posteriori energy $U^{\prime}(F / d)$. They allow also to satisfy the region continuity constraint. For the latter problem, if we assume that the horizontal distance between two coplanar regions $R_{1}$ and $R_{2}$ is greater than $W$, labels $l_{R_{1}}$ and $l_{R_{2}}$ corresponding respectively to $R_{1}$ and $R_{2}$, cannot belong to the same set $L^{\prime}(x, y)$. For example, if the site $(x, y)$ belongs to $R_{1}$, it can not be labeled $l_{R_{2}}$, although energies $U^{\prime}\left(l_{R_{1}} / d(x, y)\right)$ and $U^{\prime}\left(l_{R_{2}} / d(x, y)\right)$ are equal.

\section{Experimentation}

Hoover et al. have proposed a dedicated framework for the evaluation of range image segmentation algorithms [8], which has been used in several related works 11 1341]. The framework consists of a set of real range images, and a set of objective performance metrics. It allows to compare a machine-generated segmentation (MS) with a manually-generated segmentation, supposed ideal and representing the ground truth $(\mathrm{GT})$. Instances of region detection are classified into Correct detection, over-segmentation (one region broken is several small regions), under-segmentation (several regions merged in one large region), missed region and noise region. Region classification is performed according to a compare tool tolerance $T ; 50 \%<T \leq 100 \%$ which reflects the strictness of the classification. The 40 real images of $\mathrm{ABW}$ set are divided into two subsets: 10 training images, and 30 test images. In this study, four methods, namely USF, WSU, UB and UE, cited in [8] are involved in the result comparison.

\subsection{Parameter Selection}

Since the evaluation framework provides a set of training images with ground truth segmentation (GT), we have opted to a supervised approach for the estimation of parameters.

For the proposed method, named EBR for Edge Bayesian Regularization, five parameters should be fixed: $\operatorname{Tr}_{\theta}, \operatorname{Tr}_{h}, W, Q$, and $\zeta$. The performance criterion used in parameter selection is the average number of correctly detected regions with the compare tool tolerance $T$ set to $80 \%$. The parameters are divided into two subsets: 1) $\operatorname{Tr}_{\theta}, \operatorname{Tr}_{h}, W$ and $Q$ which represent respectively the angle threshold, the depth threshold, the window size, and the seed quality threshold. These parameters are used by the randomized region growing algorithm. 2) The potential parameter $\zeta$, used during the segmentation refinement. For the first 
parameter subset, 256 combinations namely $\left(\operatorname{Tr}_{\theta}, \operatorname{Tr}_{h}, W, Q\right) \in\{15,18,21,24\} \times$ $\{12,16,20,24\} \times\{5,7,9,11\} \times\{0.90,0.95,0.97,0.99\}$, were run on the training images. Resulting optimal values of the parameters are as follows: $\operatorname{Tr}_{\theta}=21^{\circ}$, $T r_{h}=16, W=7$ and $Q=0.97$. The selected value of $W$ permits to estimate the plane equation by considering a wide neighborhood ( $W^{2}$ pixels), whereas $Q$ ensure that the plane parameters are reliable, and the window $W \times W$ is not located between two different regions.

We have used the Coding method 2] to estimate the parameter $\zeta$. For each image in the training set, a pair of values of these parameters is calculated. One value of this parameter is calculated for each image in the training set. The average is then computed and used as the final parameter value. The optimal value for each training image is calculated by the simulated annealing algorithm 12, using a Gibbs sampler [7]. The average value of $\zeta$ obtained with the training set is $-0.587 \times 10^{-4}$.

\subsection{Experimental Results}

Region growing by randomized region seed sampling has provided better results, compared to deterministic region growing (Fig. 2b,c). However, the resulting segmentation often remains unsatisfactory. In Fig. 2r, we can note that most of the unlabeled pixels are situated on the region boundaries. Fig. $2 \mathrm{~d}$ show the result obtained after edge regularization. Many pixels of boundaries were assigned to certain regions so that resulting edges are more locally smooth.

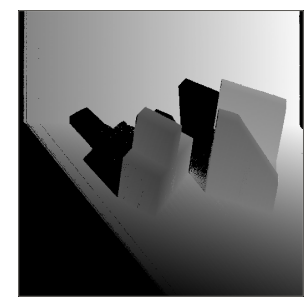

(a)

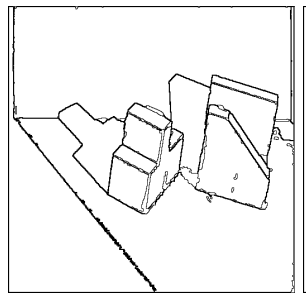

(b)

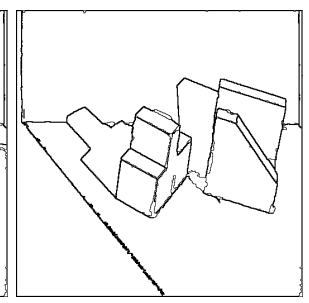

(c)

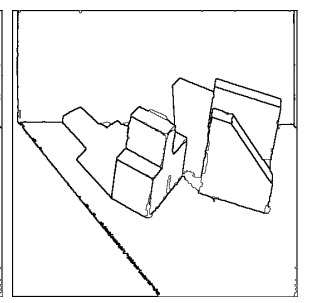

(d)

Fig. 2. Result comparison with abw.test.6 image. (a) Range image; (b) Segmentation result by deterministic region growing; (c) Segmentation result by randomized region growing; (d) Segmentation result after edge regularization.

Fig. 3illustrate the impact of the Bayesian edge regularization on the segmentation results, with all the test images. The two graphs show that segmentation results are significantly improved for the high values of the compare tool tolerance $\mathrm{T}$. Indeed, the edge regularization in range images allows to improve segmentation accuracy, by optimal labeling of pixels close to region boundaries. It was reported that segmentation methods provide better results when they pay particular attention to process region boundaries [810]. 


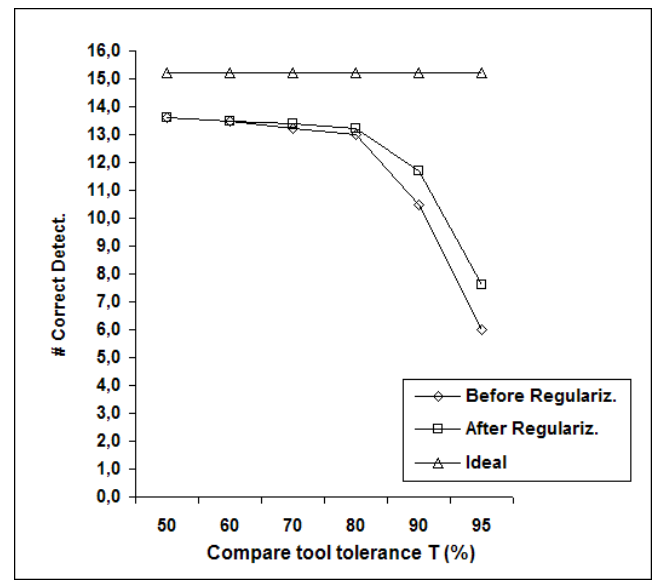

Fig. 3. Comparison of average results before and after edge regularization of the test images, according to $T ; 0.5<T \leq 1.0$

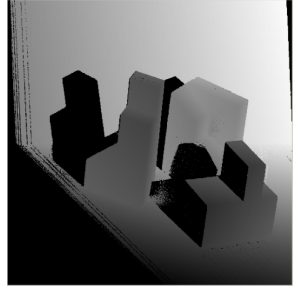

(a)

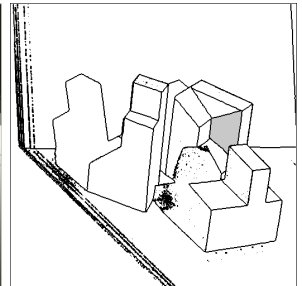

(b)

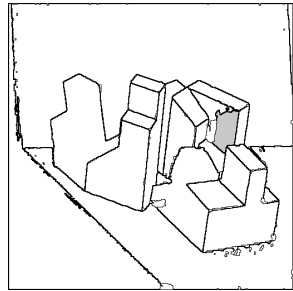

(c)

Fig. 4. Segmentation result of abw.test.8 image. (a) Range image; (b) Ground truth segmentation; (c) Segmentation result after edge regularization.

Fig. 4] shows the segmentation result of the image abw.test.8, with the compare tool tolerance $T$ set to $80 \%$. This image was considered as a typical image to compare the involved methods 8,4]. Fig. 4a shows the range image; Fig. 4b shows the ground truth segmentation, whereas 4 represents the segmentation result obtained by our method. Metrics in Table 1 show that all image regions detected by the best-referenced segmenter (UE) were detected by our method. The shadowed region has not been detected by any segmenter, due to high distortions in this region. The incorrectly detected regions are those with small sizes and situated on the horizontal support. Compared to the other methods, values of incorrect detection metrics are also good. Our method is equivalent to UE and scored higher than the others.

Fig. 5 shows the average numbers of correctly detected regions for all test images and according to the compare tool tolerance $T ; T \in\{51,60,70,80,90,95 \%\}$. Results show that the number of correctly detected regions by our method is equivalent to UE and better than those of USF, UB, and WSU. For instance, 
Table 1. Comparison results with abw.test. 8 image for $T=80 \%$

\begin{tabular}{ccccccc}
\hline Method & $\begin{array}{c}\text { GT } \\
\text { region }\end{array}$ & $\begin{array}{c}\text { Correct } \\
\text { detection }\end{array}$ & $\begin{array}{c}\text { Over- } \\
\text { Oegmentation }\end{array}$ & $\begin{array}{c}\text { Under- } \\
\text { segmentation }\end{array}$ & Missed Noise \\
\hline USF & 21 & 17 & 0 & 0 & 4 & 3 \\
WSU & 21 & 12 & 1 & 1 & 6 & 4 \\
UB & 21 & 16 & 2 & 0 & 3 & 6 \\
UE & 21 & 18 & 1 & 0 & 2 & 2 \\
EBR & 21 & 18 & 2 & 0 & 1 & 1 \\
\hline
\end{tabular}

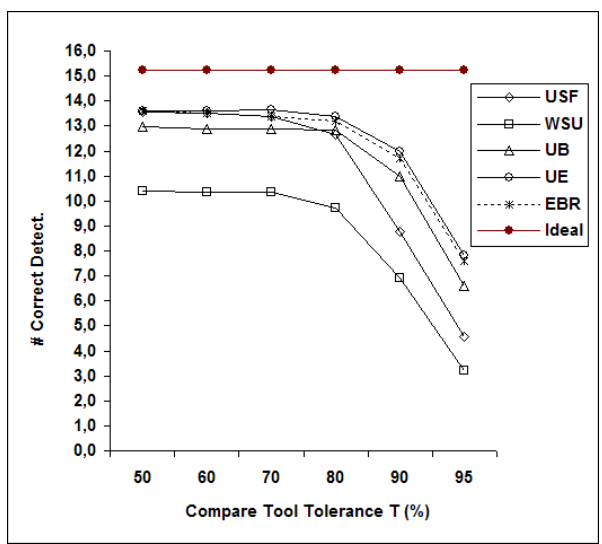

Fig. 5. Average results of correctly detected regions of all methods, according to the compare tool tolerance $T ; 0.5<T \leq 1.0$

our system scored higher than WSU and UB for all the values of the compare tool tolerance $T$. It scored higher than USF for $T \geq 60 \%$. For all incorrect detection metrics (Over-segmentation, Under-segmentation, Missed, Noise), our method has equivalent scores to those of UE and USF. The two latter scored higher than UB and WSU.

\section{Conclusion}

We have presented in this paper a new Bayesian method for range image segmentation. The refinement of the initial segmentation using the Bayesian-MRF framework has allowed improving significantly the segmentation results. We have presented a new MRF model which allows to model the edge smoothness, considered as the prior assumption on region boundaries in range images. Several tests were performed on real images from the ABW database. The average run time is 8 sec., on a Compaq $\mathrm{PC} \mathrm{n} \times 8220$. The recorded run times were better than those provided by region based methods, and equivalent to edge-based ones. For instance, the average run time for UE algorithm was $6.3 \mathrm{~min}$. Obtained results show the great potential of the proposed method for providing efficient and 
accurate range image segmentation. The proposed method scored equivalent results to those of UE algorithm, however, computing time in our case was highly improved.

\section{References}

1. Bab Hadiashar, A., Gheissari, N.: Range image segmentation using surface selection criterion. IEEE Transactions on Image Processing 15(7), 2006-2018 (2006)

2. Besag, J.E.: Spatial interaction and statistical analysis of lattice systems. Journal of the Royal Statistical Society, Series B 36, 192-236 (1974)

3. Besag, J.E.: On the statistical analysis of dirty pictures. Journal of the Royal Statistical Society, Series B 48, 259-302 (1986)

4. Ding, Y., Ping, X., Hu, M., Wang, D.: Range image segmentation based on randomized hough transform. Pattern Recognition Letters 26(13), 2033-2041 (2005)

5. Fan, T.J., Medioni, G.G., Nevatia, R.: Segmented description of 3-D surfaces. IEEE Journal of Robotics and Automation 3(6), 527-538 (1987)

6. Fischler, M.A., Bolles, R.C.: Random sample consensus: a paradigm for model fitting with applications to image analysis and automated cartography. Readings in computer vision: issues, problems, and paradigms, pp. 726-740 (1987)

7. Geman, S., Geman, D.: Stochastic relaxation, Gibbs distributions, and the Bayesian restoration of images. IEEE Transactions on Pattern Analysis and Machine Intelligence 6(6), 721-741 (1984)

8. Hoover, A., Jean-Baptiste, G., Jiang, X., Flynn, P.J., Bunke, H., Goldgof, D.B., Bowyer, K.W., Eggert, D.W., Fitzgibbon, A.W., Fisher, R.B.: An experimental comparison of range image segmentation algorithms. IEEE Transactions on Pattern Analysis and Machine Intelligence 18(7), 673-689 (1996)

9. Jain, A.K., Nadabar, S.G.: MRF model-based segmentation of range images. In: International Conference on Computer Vision, pp. 667-671, Osaka (1990)

10. Jiang, X., Bowyer, K.W., Morioka, Y., Hiura, S., Sato, K., Inokuchi, S., Bock, M., Guerra, C., Loke, R.E., Hans du Buf, J.M.: Some further results of experimental comparison of range image segmentation algorithms. In: International Conference on Pattern Recognition, vol. 4, pp. 4877-4882 (2000)

11. Jiang, X., Bunke, H.: Edge detection in range images based on Scan Line approximation. Computer Vision and Image Understanding 73(2), 183-199 (1999)

12. Kirkpatrick, S., Gelatt, C.D., Vecchi, M.P.: Optimization by simulated annealing. Readings in Computer Vision: Issues, Problems, Principles, and Paradigms, pp. 606-615 (1987)

13. Li, S., Zhao, D.: Gradient-based polyhedral segmentation for range images. Pattern Recognition Letters 24(12), 2069-2077 (2003)

14. Li, S.Z.: Markov random field modeling in image analysis. Springer-Verlag, New York, Inc. Secaucus, NJ, USA (2001)

15. Wang, X., Wang, H.: Markov random field modeled range image segmentation. Pattern Recognition Letters 25(3), 367-375 (2004) 